\title{
Zur Integration heuristischer Managementstrategien in die Dynamische Finanzanalyse
}

\author{
Von Martin Eling, Thomas Parnitzke und \\ $\mathrm{H}$ a t o S ch mei s e r, St. Gallen*
}

\section{Inhaltsverzeichnis}

1. Einleitung

2. Modellrahmen

3. Managementstrategien und ihr Einfluss auf die Performance von Versicherungsunternehmen

3.1. Managementstrategien: Ein Überblick

3.2. Performancemessung

3.3. Simulationsbeispiel

4. Fazit

\section{Einleitung}

Vor dem Hintergrund erheblicher Veränderungen im Wettbewerbsverhalten, in den Kapitalmarktbedingungen und im Aufsichtsrecht, hat die ganzheitliche Analyse der Anlagen und Verbindlichkeiten eines Versicherungsunternehmens in den vergangenen Jahren zunehmend an Bedeutung gewonnen. Ein wichtiges Instrument zur integrierten Steuerung von Aktiva und Passiva stellt die Dynamische Finanzanalyse (DFA) dar. Die DFA ist ein finanzwirtschaftlich orientierter Ansatz zur Evaluation der Hauptrisikofaktoren und ihrer Auswirkungen auf die Performance und Risikosituation des Versicherers. Dabei erfolgt eine Modellierung des Unternehmens aus der Makroperspektive, um mit Hilfe einer Simulation eine Prognose der zukünftigen Finanzlage erstellen zu können.

Die Diskussionen in der Europäischen Union bzgl. neuer Solvabilitätsvorschriften (Solvency II) und die Verwendung der DFA im Zusammenhang mit internen Risikosteuerungsmodellen haben gezeigt, dass die DFA bereits heute ein wichtiges Instrument zur Entscheidungsunterstützung in Ver-

* Dr. Martin Eling, Dipl.-Kfm. Thomas Parnitzke und Prof. Dr. Hato Schmeiser, Universität St. Gallen, Institut für Versicherungswirtschaft. Wir bedanken uns bei den Teilnehmern der Jahrestagung 2007 des Deutschen Vereins für Versicherungswissenschaft für zahlreiche Anmerkungen und Hinweise. 
sicherungsunternehmen ist. Dies gilt in besonderem Maße für den Schaden- / Unfall- und Rückversicherungsbereich. ${ }^{1}$

Trotz der zunehmenden Verbreitung von stochastischen Modellen in der Praxis und zahlreicher Abhandlungen zur DFA in der wissenschaftlichen Literatur $^{2}$ gibt es weiterhin verschiedene Aspekte in der Implementierung der DFA, die bislang nicht im Fokus der wissenschaftlichen Betrachtung standen. Einer dieser Aspekte ist die Integration von Managementstrategien mit Hilfe von spezifischen Entscheidungsregeln in DFA-Modelle. Zwar wurde dieser Aspekt regelmäßig als ein wesentlicher Schritt zur Verbesserung der Dynamischen Finanzanalyse genannt, ${ }^{3}$ konkrete Vorschläge zur Einbindung existieren bisher jedoch noch nicht.

Das Ziel dieser Arbeit besteht darin, unterschiedliche Managementstrategien in ein DFA-Modell zu integrieren und die Effekte dieser Strategien auf die Performance eines Versicherers zu analysieren. Die vorliegende Analyse baut auf der Untersuchung von Eling/Parnitzke/Schmeiser (2007) auf, welche zugleich Gegenstand des Vortrags von Martin Eling auf der Jahrestagung des Deutschen Vereins für Versicherungswissenschaft 2007 in Stuttgart war. Dabei wollen wir in diesem Beitrag zwei Aspekte, die von Teilnehmern der Jahrestagung angeregt wurden, aufgreifen und in unseren Modellrahmen integrieren. Demnach erweitern wir die Resultate der Untersuchung um zusätzliche Modellvarianten und neue Beispielsimulationen.

Der vorliegende Beitrag gliedert sich wie folgt: In Abschnitt 2 beschreiben wir den zugrunde liegenden Modellrahmen. In Abschnitt 3 werden drei Managementstrategien und ihr Einfluss auf die Performance eines Versicherungsunternehmens mittels einer Beispielsimulation analysiert. Die Arbeit schließt mit einem Fazit in Abschnitt 4.

\section{Modellrahmen}

Der Ausgangspunkt unserer Betrachtung ist ein vereinfachtes Modell eines Schaden-/Unfallversicherers. ${ }^{4}$ Wir bezeichnen mit $E C_{t}$ das Eigenkapital des Versicherers zum Zeitpunkt $t$ und mit $E_{t}$ den Gewinn des Versicherers in $t$. Damit lässt sich folgende Basisrelation für die Entwicklung des Eigenkapitals ableiten:

1 Für einen Überblick siehe Blum, P. / Dacorogna, M. (2004).

2 Vgl. grundlegend Casualty Actuarial Society (1999), Lowe, S.P./Stanard, J.N. (1997) und Kaufmann, R. / Gadmer, A. / Klett, R. (2001).

3 Vgl. z. B. D’Arcy, S.P. et al. (1997), S. 11-12, Blum, P. / Dacorogna, M. (2004), S. 518.

4 Für eine vollständige Darstellung der Modelldetails verweisen wir auf Eling, M. / Parnitzke, T. / Schmeiser, H. (2007). 


$$
E C_{t}=E C_{t-1}+E_{t}
$$

Das Eigenkapital in $t$ ergibt sich demnach als das Eigenkapital der Vorperiode $t-1$ zuzüglich des Gewinns in $t$. Der Gewinn setzt sich aus dem Kapitalanlageergebnis $I_{t}$ und dem versicherungstechnischen Ergebnis $U_{t}$ zusammen. Steuern werden im Fall eines positiven Gewinns bezahlt (dabei bezeichnet $t r$ den Steuersatz). In formaler Hinsicht ergibt sich damit:

$$
E_{t}=I_{t}+U_{t}-\max \left(\operatorname{tr} \cdot\left(I_{t}+U_{t}\right), 0\right) .
$$

Auf der Kapitalanlageseite betrachten wir riskante Anlagen (z. B. Aktien) mit der Rendite $r_{1 t}$ und sichere Anlagen (z. B. Anleihen) mit einer Rendite $r_{2 t}$. Der Anteil der riskanten Anlagen wird mit $\alpha$ bezeichnet, so dass wir die Portfoliorendite des Unternehmens $r_{p t}$ berechnen können, indem wir die Anlagerenditen $r_{1 t}$ und $r_{2 t}$ mit $\alpha$ bzw. $(1-\alpha)$ gewichten:

$$
r_{p t}=\alpha_{t-1} \cdot r_{1 t}+\left(1-\alpha_{t-1}\right) \cdot r_{2 t}
$$

Das Kapitalanlageergebnis des Unternehmens kann berechnet werden, indem die Portfoliorendite mit den zur Anlage zur Verfügung stehenden Mitteln $A_{t-1}$ multipliziert wird: ${ }^{5}$

$$
I_{t}=r_{p t} \cdot A_{t-1}
$$

Der zweite Teil des Unternehmensgewinns wird aus dem versicherungstechnischen Ergebnis generiert. Das versicherungstechnische Ergebnis ergibt sich aus den Prämieneinnahmen $P_{t-1}$ (diese werden zum Jahresanfang zahlungswirksam) abzüglich von (in $t$ zahlungswirksamen) Schäden $C_{t}$, Vertriebskosten $E x_{t-1}^{P}$ und Schadenbearbeitungskosten $E x_{t}^{C}$ :

$$
U_{t}=P_{t-1}-C_{t}-E x_{t-1}^{P}-E x_{t}^{C} .
$$

Wir nehmen für unser Versicherungsunternehmen ein relevantes Marktvolumen $M V$ an. Das Prämieneinkommen des Versicherers kann dabei als Anteil dieses Marktvolumens ermittelt werden. In unserem Modell gibt es drei Faktoren, welche die Relation zwischen dem Marktvolumen und dem Prämieneinkommen beeinflussen können. Der erste Faktor cr beschreibt den Einfluss des Sicherheitsniveaus des Versicherungsunternehmens auf die Bereitschaft der Versicherungsnehmer eine bestimmte Prämienhöhe für ein Risiko zu akzeptieren. ${ }^{6}$ Der zweite Faktor $\pi$ spiegelt den Einfluss der

5 Die für die Kapitalanlage verfügbaren Mittel $A_{t-1}$ ergeben sich aus dem Eigenkapital $E C_{t-1}$ und den Prämieneinnahmen $P_{t-1}$ abzüglich der Vertriebskosten $E x_{t-1}^{P}$.

6 Empirische Untersuchungen zeigen, dass schon eine geringe Erhöhung des Ausfallrisikos zu einer starken Abnahme der Zahlungsbereitschaft seitens der Versiche- 
aktuellen Phase des Marktzyklus wieder. Der dritte Faktor $\beta$ ist der Marktanteil, der durch Aktionen des Managements beeinflusst werden kann:

$$
P_{t-1}=c r_{t-1}^{E C_{t-1}} \cdot \pi_{t-1}^{s} \cdot \beta_{t-1} \cdot M V
$$

Damit gibt es in unserem Modellrahmen zwei Parameter, die das Management zu Beginn jeder Periode in einem bestimmten Umfang verändern kann: Der erste Parameter ist der Anteil der riskanten Anlagen $\alpha$ und der zweite Parameter ist der Marktanteil am Versicherungsmarkt, bezeichnet $\operatorname{mit} \beta$.

\section{Managementstrategien und ihr Einfluss auf die Performance von Versicherungsunternehmen}

\subsection{Managementstrategien: Ein Überblick}

Die drei Managementstrategien, welche beispielhaft in dieser Arbeit betrachtet werden, stellen mit Hilfe definierter Regeln Reaktionen des Managements auf die aktuelle Finanzlage des Unternehmens dar.

- Strategie 1 („Solvenz“): Wenn $E C_{t}$ kleiner als $M C R_{t} \cdot 1,5$ ist, wird $\alpha$ und $\beta$ um 0,05 gesenkt

Die Solvenzstrategie ist eine Risikoreduktionsstrategie. $\mathrm{Zu}$ jedem Zeitpunkt $(t=1, \ldots, T-1)$ werden $\alpha$ und $\beta$ um 0,05 reduziert, sobald das Eigenkapital unterhalb eines kritischen Wertes sinkt. Als kritischen Wert nehmen wir in dieser Arbeit beispielhaft die nach den Solvency-I-Regeln für Schaden-/Unfallversicherungsunternehmen ermittelte Soll-Solvabilität (Minimum Capital Required $(\mathrm{MCR}))^{7}$ zuzüglich eines Sicherheitszuschlags von $50 \%$ an.

- Strategie 2 („,Risiko“): Wenn $E C_{t}$ kleiner als $M C R_{t} \cdot 1,5$ ist, wird $\alpha$ und $\beta$ um 0,05 angehoben

Vor dem Hintergrund der beschränkten Haftung von Versicherungsaktiengesellschaften ist es vorstellbar, dass das Management im Falle einer schwierigen Finanzlage das Risiko nicht reduziert, sondern vielmehr erhöht. ${ }^{8}$ Demnach stellt die Risikostrategie das genaue Gegenteil der Solvenzstrategie dar.

rungsnehmer führt. Vgl. hierzu grundlegend Wakker, P.P./Thaler, R.H./Tversky, A. (1997).

7 Vgl. hierzu Farny (2006), S. 777-798.

8 Unter bestimmten Bedingungen kann es für die begrenzt haftenden Eigentümer eines Versicherungsunternehmens zielführend sein, sich risikofreudig zu verhalten. Vgl. dazu z. B. Gollier, C. / Koehl, P.-F. / Rochet, J.-C. (1997) und Doherty, N.A. (2000), S. 555-561. In der Praxis sind allerdings aufsichtsrechtliche Restriktionen zu beachten, die eine beliebige Erhöhung des Risikos verhindern sollen. 
- Strategie 3 („Wachstum“): Wenn $E C_{t}$ kleiner als $M C R_{t} \cdot 1,5$ ist, wird und $\beta$ um 0,05 gesenkt. Anderenfalls wird $\beta$ um 0,05 angehoben

Die Wachstumsstrategie kombiniert die Solvenzstrategie mit einem Wachstumsziel für das Versicherungsgeschäft. Für den Fall, dass das Eigenkapital unterhalb des kritischen Levels ist, gelten dieselben Regeln wie bei der Solvenzstrategie. Wenn sich das Eigenkapital oberhalb des kritischen Levels bewegt, nehmen wir eine Veränderung für $\beta$ in Höhe von 0,05 an.

In diesem Beitrag werden diese drei Basisstrategien um zwei Varianten erweitert. Die erste Variante bezieht sich auf eine getrennte Anpassung der Parameter $\alpha$ und $\beta$ während die zweite Variante die Zeichnungspolitik des Versicherungsunternehmens betrifft.

- Variante 1: Wenn $E C_{t}$ kleiner als $M C R_{t} \cdot 1,5$ und $I_{t}$ kleiner als $U_{t}$ ist (bzw. $I_{t}$ größer als $U_{t}$ ist), wird $\alpha$ um 0,05 gesenkt (bzw. $\beta$ um 0,05 gesenkt)

In Variante 1 werden im Falle einer negativen Entwicklung des Eigenkapitals nicht beide Parameter $\alpha$ und $\beta$ angepasst, sondern nur der Parameter $\alpha$ oder der Parameter $\beta$. Wenn die negative Entwicklung vor allem von der Kapitalanlageseite resultiert (d. h. wenn das Kapitalanlageergebnis kleiner als das versicherungstechnische Ergebnis ist), wird der Parameter $\alpha$ um 0,05 reduziert. Resultiert die negative Entwicklung dagegen vor allem aus der Versicherungstechnik, soll der Parameter $\beta$ um 0,05 verringert werden. Für die Risikostrategie implementieren wir entsprechend ein entgegengesetztes Vorgehen, d. h. beispielsweise, dass $\alpha$ erhöht wird, wenn $E C_{t}<M C R_{t} \cdot 1,5$ ist und das Kapitalanlageergebnis der letzten Periode besser als das versicherungstechnische Ergebnis war. ${ }^{9}$

- Variante 2: Wenn $\beta$ um 0,05 zunimmt, dann nimmt $E\left(C_{t}\right)$ um 0,01 zu

Wenn im Rahmen der Risiko- und der Wachstumsstrategie der Marktanteil deutlich gesteigert wird, geht dies mit einer Verschlechterung der gezeichneten Risiken einher. Eine mögliche Ursache dafür können Anpassungen der eigenen Zeichnungsregeln und -limiten sein, die notwendig werden, um die sich selbst gesteckten Wachstumsziele zu erreichen. Dementsprechend nehmen wir für den Fall einer Anhebung des Parameters $\beta$ um 0,05 eine Erhöhung des Schadenerwartungswertes um einen Prozentpunkt an. Für die Wachstumsstrategie nehmen wir im Falle einer Reduktion des Marktanteils keine Verringerung des Schadenerwartungswertes vor. ${ }^{10}$

9 Wir bedanken uns bei Herrn Prof. Dr. Andreas Richter, Ludwig-MaximiliansUniversität München, für diesen Vorschlag.

10 Wir bedanken uns bei Herrn Prof. Dr. Jochen Zimmermann, Universität Bremen, für diese Anregung. 


\subsection{Performancemessung}

Im Folgenden sollen Rendite, Risiko und Performance der Managementstrategien anhand von acht Kennzahlen untersucht werden. Diese Kennzahlen werden in Tabelle 1 vorgestellt. Eine formale Darstellung der verwendeten Maße ist in Eling / Parnitzke / Schmeiser (2007) zu finden.

\section{Tabelle 1}

Rendite-, Risiko- und Performancemaße

\begin{tabular}{|c|c|c|c|}
\hline & Symbol & $\mathrm{Maß}$ & Interpretation \\
\hline \multirow[t]{2}{*}{ Rendite } & $E(G)$ & Erwarteter Gewinn pro Jahr & Absolute Rendite \\
\hline & $R O I$ & $\begin{array}{l}\text { Erwarteter Return on } \\
\text { Investment pro Jahr }\end{array}$ & Relative Rendite \\
\hline \multirow[t]{3}{*}{ Risiko } & $\sigma(G)$ & $\begin{array}{l}\text { Standardabweichung } \\
\text { des Gewinns pro Jahr }\end{array}$ & Gesamtrisiko \\
\hline & $R P$ & Ruinwahrscheinlichkeit & Verlustrisiko \\
\hline & $E P D$ & Expected Policyholder Deficit & Erwarteter Verlust \\
\hline \multirow[t]{3}{*}{ Performance } & $S R_{\sigma}$ & Sharpe Ratio & Rendite/Gesamtrisiko \\
\hline & $S R_{R P}$ & Modifizierte Sharpe Ratio $(R P)$ & Rendite/Verlustrisiko \\
\hline & $S R_{E P D}$ & $\begin{array}{l}\text { Modifizierte Sharpe Ratio } \\
\qquad(E P D)\end{array}$ & Rendite/Erw. Verlust \\
\hline
\end{tabular}

Wir betrachten zunächst zwei Maße für die Rendite der Strategien: Der erwartete Gewinn pro Jahr ist dabei ein absolutes Renditemaß, während der Return on Investment den erwarteten Gewinn ins Verhältnis zum eingesetzten Kapitel setzt und demnach ein relatives Renditemaß darstellt.

Des Weiteren analysieren wir drei Risikomaße: Die Standardabweichung berücksichtigt sowohl positive als auch negative Abweichungen vom Erwartungswert des Gewinns. Demgegenüber betrachten die Ruinwahrscheinlichkeit (Verlustrisiko) und der „Expected Policyholder Deficit“ (erwarteter Verlust aus Sicht der Versicherungsnehmer) ausschließlich Downside-Risiken. ${ }^{11}$

Schließlich betrachten wir drei Performancemaße, welche die Rendite ins Verhältnis zum Risiko setzen. Das bekannteste Performancemaß ist dabei die Sharpe Ratio, welche die Rendite in Relation zur Standardabweichung abbildet. Da Risiko im Versicherungsbereich aber häufig auf Basis des Verlustrisikos analysiert wird, betrachten wir zusätzlich zwei Modifikationen der Sharpe Ratio, bei denen die Ruinwahrscheinlichkeit bzw. der erwartete

11 Vgl. hierzu auch Albrecht, P. / Maurer, R. (2005), S. 113-119. 
Verlust der Versicherungsnehmer (EPD) in den Nenner der Kennziffer eingehen.

\subsection{Simulationsbeispiel}

Ausgehend von einem Zeithorizont von $T=5$ Jahre können Entscheidungen bezüglich der Parameter $\alpha$ und $\beta$ zu Beginn jeder Periode getroffen werden. Wir betrachten normalverteilte Kapitalmarktrenditen; die stetige Verzinsung per annum hat einen Erwartungswert von $9 \%$ mit einer Standardabweichung von $18 \%$ im Fall der riskanten Anlagen und einen Erwartungswert von $5 \%$ mit einer Standardabweichung von $5 \%$ für die sicheren Anlagen. Da deutsche Schadenversicherungsunternehmen typischerweise etwa $40 \%$ in relativ riskante Anlagen investieren, wird $\alpha_{0}=0,40$ gesetzt. $^{12}$ Der risikolose Zinssatz $r_{f}$ (stetig, per annum, flache Zinsstruktur) beträgt $3 \%$.

Wir modellieren die Schadenquoten auf Basis einer Lognormalverteilung mit einem Erwartungswert von $86 \%$ und einer Standardabweichung von $8,6 \%$. Die Schadenabwicklungskosten betragen dabei $5 \%$ der Schadensumme $E x_{t}^{C}=0,05 \cdot C_{t}$ ), während die Vertriebskosten sich aus $E x_{t-1}^{P}=0,05 \cdot \beta_{t-1} \cdot M V+0,001 \cdot\left(\left(\beta_{t-1}-\beta_{t-2}\right) \cdot M V\right)^{2} \quad$ ergeben. Demnach fallen $5 \%$ der Kosten in Abhängigkeit vom gezeichneten Marktvolumen an, wobei sich weitere Kosten ergeben, wenn das Marktvolumen verändert wird (z. B. für Marketing- und Akquisitionsmaßnahmen).

Der Marktzyklus weist drei Zustände auf. In einer guten Marktumgebung (Zustand 1) kann ein höheres Prämieneinkommen bei einem gegebenen Marktvolumen realisiert werden. Dementsprechend wird das Prämieneinkommen mit dem Faktor $\pi=1,05$ multipliziert. In einer normalen Marktumgebung (Zustand 2) ist dieser Faktor 1 und in einer schlechten Marktumgebung 0,95. Die Wahrscheinlichkeiten für den Wechsel von einem Zustand zum Nächsten können der folgenden Matrix entnommen werden. Beispielsweise zeigt die erste Zeile, dass ausgehend von einer guten Marktumgebung (Zustand 1) die Wahrscheinlichkeit in einer guten Marktumgebung zu bleiben 0,3 ist, die Wahrscheinlichkeit in den Zustand $2 \mathrm{zu}$ wechseln 0,5 beträgt und die Wahrscheinlichkeit in den Zustand 3 zu wechseln folglich 0,2 ist:

$$
p_{s j}=\left(\begin{array}{ccc}
0,3 & 0,5 & 0,2 \\
0,2 & 0,6 & 0,2 \\
0,2 & 0,5 & 0,3
\end{array}\right) .
$$

12 Vgl. BaFin (2005), Tabelle 510. 
Das relevante Marktvolumen (d. h., $\beta=1$ ) beträgt in unserem Beispiel 200 Millionen $€$, wobei der Versicherer in $t=0$ einen Marktanteil von $\beta_{0}=0,20$ besitzt. Der Faktor cr, der die Reaktion der Kunden auf das Sicherheitsniveau beschreibt, beträgt $1(0,95)$, wenn das Eigenkapital zu Beginn der Periode oberhalb (unterhalb) der Soll-Solvabilität (MCR) liegt. Für den Steuersatz gilt $t r=25 \%$.

Die vorstehenden Inputdaten wurden gegenüber den in Eling / Parnitzke / Schmeiser (2007) verwendeten Werten leicht angepasst. Die vorgenommene Konfiguration stellt dabei eine veränderte ökonomische Situation für das Versicherungsunternehmen dar, um zusätzliche numerische Ergebnisse für ein weiteres Basisszenario zu ermitteln. ${ }^{13}$ Im Vergleich der hier betrachteten Simulationsergebnisse mit den Ergebnissen aus Eling / Parnitzke / Schmeiser (2007) lässt sich festhalten, dass bereits diese relativ geringe Anpassung des Basisszenarios eine sichtbare Veränderung in der erwarteten Rendite und den Risikomaßen bewirkt.

In der Tabelle 2 werden die Simulationsergebnisse für die verschiedenen Strategien auf Basis einer Latin-Hypercube-Simulation mit 100.000 Iterationen dargestellt. ${ }^{14}$ In einem ersten Schritt wollen wir die Ergebnisse der Solvenzstrategie mit den Ergebnissen, die auf Basis einer Simulation ohne der Implementierung einer Strategie beruhen, vergleichen.

Tabelle 2

Ergebnisse für die Managementstrategien

\begin{tabular}{|l|c|c|c|c|c|}
\hline \multicolumn{2}{|c|}{ Strategie } & "ohne“ & "Solvenz & "Risiko“ & "Wachstum “ \\
\hline \multirow{2}{*}{ Rendite } & $E(G)$ in Millionen $€$ & 4,82 & 4,72 & 4,94 & 6,16 \\
\cline { 2 - 6 } & $R O I$ in \% & 0,21 & 0,21 & 0,21 & 0,25 \\
\hline \multirow{4}{*}{ Risiko } & $\sigma(G)$ in Millionen $€$ & 2,64 & 2,71 & 2,67 & 3,87 \\
\cline { 2 - 6 } & $R P$ in \% & 0,31 & 0,10 & 0,88 & 0,33 \\
\cline { 2 - 6 } & $E P D$ in Millionen $€$ & 0,0060 & 0,0011 & 0,031 & 0,0061 \\
\hline Performance & $S R_{\sigma}$ & 1,82 & 1,74 & 1,85 & 1,59 \\
\cline { 2 - 6 } & $S R_{R P}$ & 7,82 & 24,32 & 2,81 & 9,47 \\
\cline { 2 - 6 } & $S R_{E P D}$ & 3,99 & 20,80 & 0,80 & 5,07 \\
\hline
\end{tabular}

13 Anpassungen erfolgten in dreierlei Hinsicht: Erstens wurde der Erwartungswert (bzw. die Standardabweichung) der Rendite der riskanten Anlageform von $10 \%$ auf $9 \%$ (von $20 \%$ auf $18 \%$ ) reduziert. Zweitens wurde der Erwartungswert (bzw. die Standardabweichung) der lognormalverteilten Schadenquoten von $85 \%$ auf $86 \%$ (von $8,5 \%$ auf $8,6 \%$ ) erhöht. Drittens wurden die Übergangswahrscheinlichkeiten (vgl. hierzu Formel (7)) in der dritten Spalte von $(0,1 ; 0,5 ; 0,4)$ auf $(0,2 ; 0,5 ; 0,3)$ angepasst.

14 Vgl. McKay, M. / Conover, W. / Beckman, R. (1979). 
Durch die Implementierung der Solvenzstrategie bleibt der erwartete Gewinn nahezu unverändert (Reduktion um rund $2 \%$ von 4,82 auf 4,72 Millionen $€$ ). Dagegen ergeben sich deutlich geringere Werte bei den Downside-Risikomaßen. Die Ruinwahrscheinlichkeit beträgt 0,10\% - also weniger als ein Drittel des Wertes, den man ohne die Implementierung einer Managementstrategie erhält. Die Solvenzstrategie vermeidet somit die meisten Insolvenzen, ohne das Renditeniveau nennenswert zu verändern. Demzufolge führt diese Strategie auch zu deutlich höheren Werten bei den Performancemaßen, die auf das Verlustrisiko abstellen. Beispielsweise beträgt der Wert von $S R_{R P} 24,32$ statt ursprünglich 7,82 . Wir können damit schlussfolgern, dass die Solvenzstrategie das Verlustrisiko nennenswert reduziert und damit einen wertvollen Schutz vor einer Insolvenz bieten kann.

Die Risikostrategie, die das genaue Gegenteil der Solvenzstrategie darstellt, resultiert entsprechend in einem konträren Rendite-/Risiko-Profil. Der erwartete Gewinn pro Jahr erhöht sich um $2 \%$ von 4,82 auf 4,94 Millionen $€$. Jedoch ist ein deutlicher Anstieg des Verlustrisikos zu beobachten. Beispielsweise ist die Ruinwahrscheinlichkeit rund dreimal höher als im Fall ohne Managementregel. Da der Anstieg des Risikos deutlich höher ausfällt als der Anstieg der Rendite, stellen sich die Performancemaße auf Grundlage des Verlustrisikos im Vergleich zu den anderen Strategien nun vergleichsweise schlecht dar.

Die Wachstumsstrategie ist im Vergleich zu den zuvor betrachteten Strategien deutlich flexibler, da hier der Parameter $\beta$ zum Ende jeder Periode (entweder nach oben oder nach unten) angepasst wird. Bei den anderen Strategien erfolgt dagegen nur eine Anpassung, wenn das Eigenkapital das vorgegebene kritische Niveau $\left(M C R_{t} \cdot 1.5\right)$ unterschreitet. Aus diesem Grund erhalten wir bei der Wachstumsstrategie ein deutlich verändertes Rendite- / Risiko-Profil. Der erwartete Gewinn pro Jahr erhöht sich um 27,8\%. Die Ruinwahrscheinlichkeit entspricht dagegen in etwa dem Wert ohne Verwendung einer Strategie. Im Vergleich zur Solvenzstrategie stellt sich die Wachstumsstrategie damit als interessante Alternative für diejenigen Manager dar, die eine höhere Rendite anstreben, allerdings auch bereit sind ein höheres Risiko zu tragen.

Im zweiten Schritt wollen wir nun Simulationsergebnisse für die zwei beschriebenen Varianten darstellen, die im Rahmen der Untersuchung von Eling / Parnitzke / Schmeiser (2007) nicht betrachtet wurden. Tabelle 3 zeigt wiederum Ergebnisse auf Basis einer Latin-Hypercube-Simulation mit 100.000 Iterationen. 
Tabelle 3

Ergebnisse für die Varianten 1 und 2

\begin{tabular}{|c|c|c|c|c|c|c|c|}
\hline \multirow{2}{*}{\multicolumn{2}{|c|}{$\begin{array}{l}\text { Varianten } \\
\text { Strategie }\end{array}$}} & \multicolumn{4}{|c|}{ Variante 1} & \multicolumn{2}{|c|}{ Variante 2} \\
\hline & & \multirow{2}{*}{$\begin{array}{c}\text { "ohne“ } \\
4,82\end{array}$} & \multirow{2}{*}{$\begin{array}{c}\text { „Solvenz" } \\
4,76\end{array}$} & \multirow{2}{*}{$\begin{array}{c}\text { „Risiko“ } \\
4,87\end{array}$} & \multirow{2}{*}{$\begin{array}{c}\text { „Wachstum“ } \\
6,22\end{array}$} & \multirow{2}{*}{$\begin{array}{c}\text { „Risiko“ } \\
4,86\end{array}$} & \multirow{2}{*}{$\begin{array}{c}\text { „Wachstum“ } \\
5,06\end{array}$} \\
\hline Rendite & $\begin{array}{c}E(G) \text { in } \\
\text { Millionen } €\end{array}$ & & & & & & \\
\hline & $R O I$ in \% & 0,21 & 0,21 & 0,21 & 0,25 & 0,21 & 0,22 \\
\hline \multirow[t]{3}{*}{ Risiko } & $\begin{array}{c}\sigma(G) \text { in } \\
\text { Millionen } €\end{array}$ & 2,64 & 2,69 & 2,65 & 3,84 & 2,75 & 3,72 \\
\hline & $\mathrm{RP}$ in \% & 0,31 & 0,14 & 0,51 & 0,43 & 1,33 & 0,57 \\
\hline & $\begin{array}{c}\text { EPD in } \\
\text { Millionen } €\end{array}$ & 0,0060 & 0,0020 & 0,0128 & 0,0082 & 0,0539 & 0,0120 \\
\hline \multirow{3}{*}{$\begin{array}{l}\text { Perfor- } \\
\text { mance }\end{array}$} & $S R_{\sigma}$ & 1,82 & 1,77 & 1,84 & 1,62 & 1,76 & 1,36 \\
\hline & $S R_{R P}$ & 7,87 & 16,86 & 4,74 & 7,26 & 1,82 & 4,48 \\
\hline & $S R_{E P D}$ & 3,99 & 12,09 & 1,90 & 3,78 & 0,45 & 2,12 \\
\hline
\end{tabular}

Bei Variante 1 werden für die drei Managementstrategien im Falle einer negativen Entwicklung des Eigenkapitals nicht beide Parameter $\alpha$ und $\beta$ angepasst, sondern nur der Parameter $\alpha$ oder der Parameter $\beta$ Im Vergleich der Simulationsergebnisse der Tabellen 2 und 3 zeigt sich, dass diese Variante zu weniger massiven Veränderungen der Rendite- und Risikomaße führt. Beispielsweise erhöht sich die Ruinwahrscheinlichkeit im Rahmen der Risikostrategie nun nicht mehr auf 0,88\%, sondern nur noch auf 0,51\%. Im Vergleich der Performancemaße zeigt sich, dass die Variante in der sowohl der Parameter $\alpha$ als auch der Parameter $\beta$ angepasst wird, bei der Solvenz- und der Wachstumsstrategie bessere Performancewerte erzielt. Bei der Risikostrategie stellt sich dagegen die Variante 1 besser dar, weil sich hier das Risiko für $E C_{t}<M C R_{t} \cdot 1,5$ weniger stark erhöht und damit ein positiver Einfluss auf die Performancewerte von $S R_{R P}$ und $S R_{E P D}$ erzielt wird. Eine interessante Weiterentwicklung dieser Variante bestände darin, Autokorrelationsbeziehungen unter den Kapitalmarktrenditen und zwischen den Schäden zu integrieren.

Für die Variante 2 wird der Erwartungswert des Schadens um einen Prozentpunkt erhöht, wenn der Parameter $\beta$ im Rahmen der Risiko- oder der Wachstumsstrategie angehoben wird. Wie aus den letzten beiden Spalten der Tabelle 3 hervorgeht, bewirkt dies vor allem bei der Wachstumsstrategie eine deutliche Veränderung der Rendite- und Risikomaße. Der erwartete Gewinn pro Jahr reduziert sich um 18,65\% von 6,22 auf 5,06 Millionen $€$. Die Ruinwahrscheinlichkeit erhöht sich dagegen von $0,43 \%$ auf $0,57 \%$. Die Verschlechterung der Zeichnungsbedingungen impliziert damit eine deutliche Reduktion der Performance des Versicherungsunterneh- 
mens: Beispielsweise verringert sich der Wert von $S R_{R P}$ um $38.29 \%$ von 7,26 auf 4,48 .

\section{Fazit}

Das Ziel dieser Arbeit bestand darin, unterschiedliche Managementstrategien in die Dynamische Finanzanalyse (DFA) zu integrieren und ihre Effekte auf die Rendite und das Risiko eines Schaden-/Unfallversicherers zu analysieren. Dabei haben wir das Modell aus Eling / Parnitzke / Schmeiser (2007) um zwei Modellvarianten erweitert und diese in Rahmen einer neuen Beispielsimulation dargestellt. Es zeigte sich, dass eine Solvenzstrategie, welche die Volatilität des Kapitalanlage- und des versicherungstechnischen Ergebnisses reduziert, eine sinnvolle Strategie im Hinblick auf die Reduktion von Verlustrisiken ist. Eine Wachstumsstrategie, welche die Solvenzstrategie mit einem Wachstumsziel für das Versicherungsgeschäft verknüpft, stellt eine interessante Alternative für diejenigen Manager dar, die ein höheres Renditeniveau anstreben, allerdings auch bereit sind, ein erhöhtes Risiko zu tragen.

Reagiert das Management auf finanzielle Schwierigkeiten allein mit einer Veränderung der Investmentpolitik oder einer Veränderung der Zeichnungspolitik im Versicherungsmarkt (Variante 1), hat dies im Rahmen unserer Beispielsimulation einen vergleichsweise geringen Einfluss auf Rendite und Risiko des Versicherungsunternehmens. Mit einem gleichzeitigen Eingriff auf die Investmentpolitik und das Versicherungsgeschäft kann das Management in einer finanziellen Notlage dagegen deutlich effizienter Einfluss auf die Risikosituation des Versicherungsunternehmens nehmen.

Verschlechtern sich mit der Ausweitung des Marktanteils die Möglichkeiten zur Zeichnung guter Risiken im Versicherungsgeschäft (Variante 2), so hat dies im Rahmen unserer Beispielsimulation überproportional starke Rückkopplungseffekte auf Rendite und Risiko des Versicherungsunternehmens zur Folge. Diese Effekte können die Attraktivität einer Markterweiterung massiv reduzieren.

Im Rahmen der Weiterentwicklung des DFA-Modells ist es interessant, Autokorrelationseffekte für die Kapitalmarktrenditen und für die Schäden zu berücksichtigen und eine Untersuchung dieser Abhängigkeitsbeziehungen auf Rendite und Risiko des Versicherungsunternehmens vorzunehmen. Die heuristischen Managementstrategien, die im Rahmen dieses DFA-Modells implementiert wurden, sollen helfen, Zusammenhänge zwischen zentralen Treibern des Versicherungsgeschäfts zu erkennen. Die vorgestellten Strategien stellen jedoch nur Beispiele aus einer Vielzahl möglicher Entscheidungskriterien dar. Eine Erweiterung könnte darin liegen, in Abhängigkeit einer konkreten Zielfunktion des Versicherers optimalen Managementstrategien zu suchen und die Optimierungsergebnisse mit den Ergeb- 
nissen der hier betrachteten heuristischen Managementregeln zu vergleichen.

\section{Literaturverzeichnis}

Albrecht, P./Maurer, R. (2005): Investment- und Risikomanagement, 2. Auflage, Stuttgart.

Blum, P. / Dacorogna, M. (2004): DFA-Dynamic Financial Analysis, in: Teugels, J./ Sundt, B. (Hrsg.): Encyclopedia of Actuarial Science, New York, S. 505-519.

BaFin (2005): Jahresbericht 2004, Statistik der Bundesanstalt für Finanzdienstleistungsaufsicht - Erstversicherungsunternehmen, Bundesanstalt für Finanzdienstleistungsaufsicht, Bonn.

Casualty Actuarial Society (1999): DFA Research Handbook, prepared by the Dynamic Financial Analysis Committee of the Casualty Actuarial Society.

D'Arcy, S.P./Gorvett, R.W./Herbers, J.A./Hettinger, T.E./Lehmann, S.G./Miller, M.J. (1997): Building a Public Access PC-Based DFA Model, Casualty Actuarial Society Forum, Summer, S. 1-40.

Doherty, N.A. (2000): Integrated Risk Management: Techniques and Strategies for Reducing Risk, New York.

Eling, M. / Parnitzke, T. / Schmeiser, H. (2007): Management Strategies and Dynamic Financial Analysis, Arbeitspapier, Universität St. Gallen, erscheint in: Variance.

Farny, D. (2006): Versicherungsbetriebslehre, 4. Auflage, Karlsruhe.

Gollier, C./Koehl, P.-F./Rochet, J.-C. (1997): Risk-Taking Behavior with Limited Liability and Risk Aversion, in: Journal of Risk and Insurance, Vol. 64, No. 2, S. $347-370$.

Kaufmann, R. / Gadmer, A. / Klett, R. (2001): Introduction to Dynamic Financial Analysis, in: ASTIN Bulletin, Vol. 31, No. 1, S. 213-249.

Lowe, S.P./Stanard, J.N. (1997): An Integrated Dynamic Financial Analysis and Decision Support System for a Property Catastrophe Reinsurer, in: ASTIN Bulletin, Vol. 27, No. 2, S. 339-371.

McKay, M./ Conover, W./Beckman, R. (1979): A Comparison of Three Methods for Selecting Values of Input Variables in the Analysis of Output from a Computer Code, in: Technometrics, Vol. 21, No. 1, S. 239-245.

Wakker, P.P. / Thaler, R.H. / Tversky, A. (1997): Probabilistic Insurance, in: Journal of Risk and Uncertainty, Vol. 15, No. 1, S. 7-28.

\section{Zusammenfassung}

Die Dynamische Finanzanalyse (DFA) hat sich in den vergangenen Jahren zu einem wichtigen Instrument zur Analyse der Finanzlage eines Versicherungsunternehmens entwickelt. Trotz der zunehmenden Verbreitung der DFA in der Praxis und zahlreicher Abhandlungen in der wissenschaftlichen Literatur gibt es weiterhin Aspekte in der Implementierung der DFA, die 
bislang nicht im Fokus wissenschaftlicher Untersuchungen standen. Einer dieser Aspekte ist die Integration von Managementstrategien in DFAModelle. Das Ziel dieser Arbeit besteht darin, Managementstrategien in ein DFA-Modell zu integrieren und ihre Effekte auf die Rendite- und die Risikoposition eines Versicherers zu analysieren. Dabei erweitern wir die Ergebnisse aus Eling / Parnitzke / Schmeiser (2007) um zwei Modellvarianten und stellen diese Varianten im Rahmen einer neuen Beispielsimulation dar.

\begin{abstract}
Dynamic financial analysis (DFA) has become an important tool in analyzing the financial situation of insurance companies. Constant development and documentation of DFA tools has occurred during the last years. However, several questions concerning the implementation of DFA systems have not been answered in the DFA literature to date. One such important issue is the consideration of management strategies in the DFA context. The aim of this paper is to study the effects of different management strategies on a non-life insurer's risk and return profile. Therefore, we extend the results of a recent working paper by Eling / Parnitzke/Schmeiser (2007) with two variants and test these variants numerically within a DFA simulation study.
\end{abstract}

